\title{
DOSES DE NITROGÊNIO E DENSIDADES DE PLANTAS COM E SEM UM REGULADOR DE CRESCIMENTO AFETANDO O TRIGO, CULTIVAR OR-1
}

\author{
NITROGEN DOSES AND PLANT DENSITIES WITH AND WITHOUT A GROWTH \\ REGULATOR AFFECTING WHEAT, CULTIVAR OR-1
}

\author{
Jeferson Zagonel ${ }^{1}$ Wilson Story Venancio ${ }^{2}$ Reni Pedro Kunz ${ }^{3}$ Humberto Tanamati ${ }^{3}$
}

RESUMO

Doses de nitrogênio e elevadas populações de plantas são utilizadas visando a obtenção de altas produtividades em trigo. Porém, estes fatores podem promover o acamamento das plantas, especialmente para as cultivares de porte médio ou alto. $O$ uso de produtos que reduzem a estatura das plantas pode minimizar este problema. Visando avaliar o efeito do regulador de crescimento trinexapc-ethyl em diferentes populações de plantas e doses de nitrogênio, na cultivar de trigo OR-1, instalou-se um experimento na Fazenda Escola "Capão da Onça", da Universidade Estadual de Ponta Grossa, em Ponta Grossa, PR, no ano de 1999. O delineamento experimental foi blocos ao acaso em esquema fatorial 2 $x 3 \times 4$, em quatro repetições. Os vinte e quatro tratamentos constaram da aplicação de $125 \mathrm{~g}$ i.a./ha de trinexapac-ethyl $e$ testemunha; densidades de 55, 75 e 112 plantas $/ m$ no espaçamento de 0,17m entre fileiras e doses de 0, 45, 90 e $135 \mathrm{~kg} / \mathrm{ha}$ de nitrogênio em cobertura. A aplicação do trinexapac-ethyl resultou em plantas com entre-nós mais curtos; em aumento do número de espigas/m e da produtividade. Com o aumento da dose de nitrogênio, ocorreu aumento da estatura das plantas, do número de espigas $/ m$ e da produtividade. Com o aumento da densidade de plantas, o diâmetro do caule, a massa seca das plantas e o número de grãos por espiga diminuíram mas o número de espigas/m e o peso de mil grãos aumentaram, sem afetarem a produtividade. Não ocorreu acamamento em nenhum dos tratamentos.

Palavras-chave: Triticum aestivum L., produtividade, trinexapac-ethyl.

SUMMARY

Elevated nitrogen rates and high plant populations are adopted with the purpose of achieving high wheat yields. However, these factors may promote the lodging of the plants, especially for the medium and tall height cultivars. The utilization of products which reduce plant height could minimize this problem. With the aim of evaluating the effects of a growth regulator on different plant populations and nitrogen rates for the wheat cultivar OR-1, an experiment was established at the UEPG "Capão da Onça" School Farm in Ponta Grossa, PR, in 1999. The experimental design was complete randomized blocks in a factorial design $2 \times 3 \times 4$ with four replications. The twenty four treatments consisted in the application of $125 \mathrm{~g}$ a.i./ha of trinexapac-ethyl and control; plant densities of 55, 75 and 112 plants $/ \mathrm{m}$ in the row spacing of $0.17 \mathrm{~m}$ and rates of $0,45,90$, and $135 \mathrm{~kg} / \mathrm{ha}$ of surface broadcasted nitrogen. The application of trinexapac-ethyl resulted in short internodes of plants, increase of the number of spikes/m and of the productivity. As the nitrogen rate increased, it also happered to plant height, number of spikes/m and yield. With the increase of plant density the stem diameter, the dry matter of plants and the number of grains/spike decreased; however, the number of spikes/m and the weight of 1000 grains increased with no effect on productivity. No lodging was observed in any of the treatments.

Key words: Triticum aestivum L., yield, trinexapac-ethyl.

\section{INTRODUÇÃO}

A cultura do trigo no Brasil vem alcançando, a cada dia, maior importância frente aos países produtores e exportadores, alicerçada nos ganhos de produtividade, na rentabilidade e na melhoria de sua qualidade industrial (EMBRAPA, 1997). A produção final da cultura é definida em função da cultivar utilizada, da quantidade de

\footnotetext{
${ }^{1}$ Engenheiro Agrônomo, Doutor, Professor Adjunto do Departamento de Fitotecnia e Fitossanidade (DEFITO) da Universidade Estadual de Ponta Grossa (UEPG), Praça Santos Andrade s/n, 84.010.790, Ponta Grossa, PR. E-mail: jzagonel@ convoy.com.br. Autor para correspondência.

${ }^{2}$ Engenheiro Agrônomo, MSc., Professor Assistente, DEFITO, UEPG.

${ }^{3}$ Engenheiro Agrônomo, Novartis Agribusiness Ltda.
} 
insumos e das técnicas de manejo empregadas. A crescente utilização de cultivares de alto potencial produtivo tem implicado no uso mais freqüente de insumos, entre os quais a adubação nitrogenada mostra-se importante na definição da produtividade.

A dose de nitrogênio a ser utilizada baseia-se na estatura das plantas e na fertilidade do solo. Em média são utilizados de 30 a $60 \mathrm{~kg} / \mathrm{ha}$ do elemento (COSTA \& OLIVEIRA, 1998), sendo as menores doses recomendadas para as cultivares de porte alto e/ou para solos de alta fertilidade. Entretanto, algumas cultivares podem responder a até $120 \mathrm{~kg} / \mathrm{ha}$ de nitrogênio (FREITAS et al., 1995; VIEIRA et al., 1995). Para o melhor aproveitamento do nitrogênio, recomenda-se o parcelamento da dose, aplicando-se parte na semeadura e parte em cobertura no final do perfilhamento (IAPAR, 1999).

Pela capacidade de emissão de perfilhos com espigas férteis, o trigo apresenta a propriedade de preencher os espaços vazios na lavoura, compensando possíveis falhas na semeadura. Outra característica da cultura é a capacidade de aumentar ou diminuir o número de espiguetas por inflorescência, de acordo com a densidade de semeadura (MUNDSTOCK, 1999). Em geral, a quantidade de sementes a ser utilizada, visa a obtenção de densidades de 300 a 400 plantas/m² (IAPAR, 1999; SEGANFREDO, 1999), sendo as menores quantidades recomendadas para solos de alta fertilidade.

Altas densidades de plantas e elevadas doses de nitrogênio são fatores positivos para o aumento da produtividade, porém, podem resultar no acamamento da cultura, o que interfere negativamente na produção e na qualidade dos grãos. O uso de redutores de crescimento é uma prática difundida com sucesso na Europa, onde o trinexapac-ethyl tem-se mostrado efetivo na redução da estatura das plantas de cereais de inverno, evitando o acamamento (AMREIN et al., 1989; KERBER $\boldsymbol{e t} \boldsymbol{a l}$. , 1989). O produto atua no balanço das giberelinas, reduzindo drasticamente os níveis da $\mathrm{GA}_{1}$, responsável pelo crescimento das plantas (DAVIES, 1987), ao mesmo tempo que aumenta acentuadamente seu precursor biosintético imediato $\mathrm{GA}_{20}$. A queda no nível do ácido giberélico ativo $\left(\mathrm{GA}_{1}\right)$ é a provável causa da inibição do crescimento das plantas (WEILER \& ADAMS, 1991).

O presente trabalho teve como objetivo avaliar os efeitos do regulador de crescimento trinexapac-ethyl em algumas características agronômicas e na produtividade da cultivar de trigo OR-1 em diferentes densidades de plantas e doses de nitrogênio.

\section{MATERIAL E MÉTODOS}

O experimento foi instalado na Fazenda Escola "Capão da Onça" da Universidade Estadual de Ponta Grossa, no município de Ponta Grossa, PR, no ano de 1999. O solo no local é um Cambissolo háplico distrófico de textura argilosa.

$\mathrm{O}$ delineamento experimental utilizado foi de blocos ao acaso, em esquema fatorial $2 \times 3 \times 4$, com quatro repetições. As parcelas apresentaram área total de $15,0 \mathrm{~m}^{2}(6,0 \times 2,5 \mathrm{~m})$ e área útil de $9,0 \mathrm{~m}^{2}$ $(5,0 \times 1,8 \mathrm{~m})$. Os tratamentos constaram da aplicação de $125 \mathrm{~g}$ i.a./ha de trinexapac-ethyl e testemunha; densidades de 55, 75 e 112 plantas/m obtidas através da semeadura de 140,210 e $280 \mathrm{~kg} /$ ha de sementes, respectivamente e doses de $0,45,90$ e $135 \mathrm{~kg} / \mathrm{ha}$ de nitrogênio na forma de uréia, aplicadas em cobertura, no perfilhamento.

A semeadura foi realizada mecanicamente no dia 17/05/99 em fileiras espaçadas de $0,17 \mathrm{~m}$, no sistema de plantio direto na palha, sobre restos de cultura de feijão. A emergência das plantas ocorreu no dia 28/05/99 e a adubação consistiu da aplicação de $300 \mathrm{~kg} / \mathrm{ha}$ de adubo de fórmula comercial $05-25$ 25 , na semeadura.

O trinexapac-ethyl foi aplicado através de pulverizador costal, à pressão constante de $30 \mathrm{lb} / \mathrm{pol}^{2}$, pressurizado por $\mathrm{CO}_{2}$ comprimido, com bicos de jato plano "leque" XR 110-015. Aplicou-se o equivalente à $200 \ell /$ ha de calda. Na aplicação, as plantas de trigo apresentavam de 3 a 4 perfilhos, com o $1^{\circ}$ e $2^{\circ}$ nós visíveis, estatura entre 30 e $35 \mathrm{~cm}$, e condições normais de desenvolvimento.

A cultivar utilizada foi OR-1, de estatura baixa $(<81 \mathrm{~cm})$, espiga fusiforme e oblonga, ereta, grãos semiduros, moderadamente resistente à germinação na espiga, ciclo de 64 a 84 dias até o espigamento e resistente ao acamamento (IAPAR, 1999). Seu ciclo total é de aproximadamente 120 dias na Região dos Campos Gerais e sua produtividade média (de 3 anos) foi de $3.666 \mathrm{~kg} / \mathrm{ha}$ (SEGANFREDO, 1999).

No florescimento, foram determinados o comprimento dos entre-nós, a estatura de plantas, a massa das plantas secas e o diâmetro do caule. $\mathrm{Na}$ fase de enchimento de grãos (massa mole) foi determinado visualmente o acamamento. Na colheita foram determinados os componentes da produção e a produtividade, sendo a última calculada através da colheita da área útil das parcelas. Os dados obtidos foram submetidos à análise de variância sendo as diferenças entre as médias comparadas pelo teste da DMS (t teste) no nível de $5 \%$ de probabilidade.

\section{RESULTADOS E DISCUSSÃO}

Ocorreram modificações em várias características estudadas, porém, os efeitos da densidade de plantas, das doses de nitrogênio e da 
aplicação do trinexapac-ethyl aconteceram em sua maioria isoladamente, uma vez que só foram observadas interações significativas entre o nitrogênio e o trinexapac-ethyl para a estatura de plantas e para o comprimento do segundo entrenó (Tabelas 1 a 3).

O trinexapac-ethyl promoveu alterações importantes tanto nas características das plantas estudadas como na produtividade e seus componentes. O produto promoveu uma redução substancial na estatura das plantas, pela diminuição do comprimento dos quatro entre-nós (Tabela 1), sem entretanto afetar o diâmetro do caule e a massa das plantas secas (Tabela 2). O resultado destas alterações foi o aparecimento de plantas mais compactas, com melhor direcionamento dos fotoassimilados para a produção de grãos, uma vez que o número de espigas por metro e de espiguetas por espiga (Tabela 3) aumentaram e determinaram maior produtividade (Tabela 4). O peso de 1000 grãos (Tabela 4) diminuiu com a aplicação de trinexapac-ethyl, mais pelo efeito clima e planta para o enchimento do maior número de grãos produzidos, do que pela aplicação do produto.

Os efeitos da redução de estatura na redução do acamamento não puderam ser observados, uma vez que o clima foi ameno, com poucos ventos, fator que somado à baixa estatura média da cultivar OR-1, implicaram na não ocorrência do acamamento, mesmo nas parcelas tratadas com as maiores doses de nitrogênio e nas mais altas populações de plantas. Mesmo assim, foi nítida a vantagem do trinexapac-ethyl na redução da estatura das plantas (Tabela 2), sugerindo que em

Tabela 1 - Comprimento dos entre-nós $(\mathrm{cm})$ do trigo em função da densidade de plantas, de doses de nitrogênio e do regulador de crescimento trinexapac-ethyl. Fazenda Escola, UEPG. Ponta Grossa - PR, 1999.

\begin{tabular}{|c|c|c|c|c|c|c|}
\hline $\begin{array}{l}\text { Densidade } \\
\text { (plantas/m) }\end{array}$ & \multicolumn{2}{|c|}{$\begin{array}{c}1^{\circ} \text { entre-nó } \\
\text { Média }\end{array}$} & \multicolumn{2}{|c|}{$\begin{array}{l}2^{\circ} \text { entre-nó } \\
\text { Média }\end{array}$} & $\begin{array}{c}3^{\circ} \text { entre-nó } \\
\text { Média }\end{array}$ & $\begin{array}{l}\text { Pedúnculo } \\
\text { Média }\end{array}$ \\
\hline 55 & \multicolumn{2}{|c|}{$6,7 \mathrm{a}$} & \multicolumn{2}{|c|}{$10,7 \mathrm{a}$} & $14,3 \mathrm{a}$ & $18,0 \mathrm{a}$ \\
\hline 75 & \multicolumn{2}{|c|}{$6,6 a$} & \multicolumn{2}{|c|}{$10,7 \mathrm{a}$} & $14,6 \mathrm{a}$ & $18,6 a$ \\
\hline 112 & \multicolumn{2}{|c|}{$7,1 \mathrm{a}$} & \multicolumn{2}{|c|}{$10,5 \mathrm{a}$} & $14,6 \mathrm{a}$ & $18,8 \mathrm{a}$ \\
\hline Nitrogênio (kg/ha) & \multicolumn{2}{|c|}{ Média } & Com & Sem & Média & Média \\
\hline 0 & \multicolumn{2}{|c|}{$6,9 a$} & $8,8 \mathrm{~b}$ & $11,6 a$ & $13,5 \mathrm{c}$ & $16,3 \mathrm{~b}$ \\
\hline 45 & \multicolumn{2}{|c|}{$6,7 a$} & $9,3 \mathrm{~b}$ & $11,6 a$ & $14,4 \mathrm{~b}$ & $18,8 \mathrm{a}$ \\
\hline 90 & \multicolumn{2}{|c|}{$7,2 \mathrm{a}$} & $10,1 \mathrm{a}$ & $11,7 \mathrm{a}$ & $14,8 \mathrm{ab}$ & $19,2 \mathrm{a}$ \\
\hline 135 & \multicolumn{2}{|c|}{$6,5 \mathrm{a}$} & $10,1 \mathrm{a}$ & $11,7 \mathrm{a}$ & $15,2 \mathrm{a}$ & $19,5 \mathrm{a}$ \\
\hline Média & Com & Sem & Com & Sem & Com & Com \\
\hline Trinexapac-ethyl & $6,1 \mathrm{~B}$ & $7,5 \mathrm{~A}$ & $9,6 \mathrm{~B}$ & $11,6 \mathrm{~A}$ & $12,1 \mathrm{~B} \quad 16,8 \mathrm{~A}$ & $18,0 \mathrm{~B} \quad 19,0 \mathrm{~A}$ \\
\hline Coef. variação & \multicolumn{2}{|c|}{$17,2 \%$} & \multicolumn{2}{|c|}{$7,2 \%$} & $7,5 \%$ & $11,4 \%$ \\
\hline
\end{tabular}

Médias seguidas da mesma letra, minúsculas nas colunas e maiúsculas na linhas, não diferem pelo teste da DMS (t teste) ao nível de $5 \%$ de probabilidade.
Tabela 2 - Estatura de plantas $(\mathrm{cm})$, massa das plantas secas $(\mathrm{g})$ e diâmetro do caule $(\mathrm{cm})$ do trigo em função da densidade de plantas, de doses de nitrogênio e do regulador de crescimento trinexapacethyl. Fazenda Escola, UEPG. Ponta Grossa, PR. 1999

\begin{tabular}{|c|c|c|c|c|c|c|}
\hline $\begin{array}{c}\text { Densidade } \\
\text { (plantas/m) }\end{array}$ & \multicolumn{2}{|c|}{$\begin{array}{l}\text { Estatura } \\
\text { Média }\end{array}$} & \multicolumn{2}{|c|}{$\begin{array}{c}\text { Massa seca } \\
\text { Média }\end{array}$} & \multicolumn{2}{|c|}{$\begin{array}{c}\text { Diâmetro do caule } \\
\text { Média }\end{array}$} \\
\hline 55 & \multicolumn{2}{|c|}{$49,0 \mathrm{a}$} & \multicolumn{2}{|c|}{$1,39 a$} & \multicolumn{2}{|c|}{$3,29 a$} \\
\hline 75 & \multicolumn{2}{|c|}{$50,0 \mathrm{a}$} & \multicolumn{2}{|c|}{$1,32 \mathrm{a}$} & \multicolumn{2}{|c|}{$3,13 \mathrm{~b}$} \\
\hline 112 & \multicolumn{2}{|c|}{$50,6 a$} & \multicolumn{2}{|c|}{$1,21 \mathrm{~b}$} & \multicolumn{2}{|c|}{$3,00 \mathrm{c}$} \\
\hline Nitrogênio $(\mathrm{kg} / \mathrm{ha})$ & Com & Sem & \multicolumn{2}{|c|}{ Média } & \multicolumn{2}{|c|}{ Média } \\
\hline 0 & $41,1 \mathrm{c}$ & $53,7 \mathrm{a}$ & \multicolumn{2}{|c|}{$1,28 \mathrm{a}$} & \multicolumn{2}{|c|}{$3,13 \mathrm{ab}$} \\
\hline 45 & $45,5 \mathrm{~b}$ & $54,7 \mathrm{a}$ & \multicolumn{2}{|c|}{$1,34 \mathrm{a}$} & \multicolumn{2}{|c|}{$3,18 \mathrm{a}$} \\
\hline 90 & $47,7 \mathrm{ab}$ & $54,0 \mathrm{a}$ & \multicolumn{2}{|c|}{$1,26 \mathrm{a}$} & \multicolumn{2}{|c|}{$3,08 \mathrm{~b}$} \\
\hline 135 & $48,0 \mathrm{a}$ & $54,3 \mathrm{a}$ & \multicolumn{2}{|c|}{$1,35 \mathrm{a}$} & \multicolumn{2}{|c|}{$3,17 \mathrm{a}$} \\
\hline Média & Com & Sem & Com & Sem & Com & Sem \\
\hline Trinexapac-ethyl & $45,6 \mathrm{~B}$ & $54,2 \mathrm{~A}$ & $1,2 \mathrm{~A}$ & $1,3 \mathrm{~A}$ & $3,1 \mathrm{~A}$ & $3,1 \mathrm{~A}$ \\
\hline Coef. variação & \multicolumn{2}{|c|}{$5,8 \%$} & \multicolumn{2}{|c|}{$13,6 \%$} & \multicolumn{2}{|c|}{$5,0 \%$} \\
\hline
\end{tabular}

Médias seguidas da mesma letra, minúsculas nas colunas e maiúsculas na linhas, não diferem pelo teste da DMS (t teste) ao nível de 5\% de probabilidade.

condições adversas de clima, o acamamento seria evitado, como foi observado por AMREIN et al. (1989). Por outro lado, mesmo sem a ocorrência do acamamento, o produto promoveu maior produtividade, atribuída ao aumento nos componentes da produção, especialmente do número de grãos, que tem correlação negativa com a estatura das plantas (GE \& ZHANG, 1990; CAVASSIM \& BORÉM, 1998).

A densidade de plantas não afetou a estatura das plantas e o comprimento dos entre-nós. $\mathrm{O}$ aumento da densidade resultou em plantas de menor massa seca e diâmetro do caule (Tabela 2), deixando-as mais suscetíveis ao acamamento. Resultou também em menor número de perfilhos por planta (Tabela 3) e, como o número de plantas por área em geral é o mesmo para uma determinada situação ou local, a quantidade de plantas emergidas irá definir o número de perfilhos a serem emitidos para compensar a falta ou o excesso destas plantas (MUNDSTOCK, 1999). Assim, o aumento do número de plantas observadas no experimento resultou na emergência de um menor número de perfilhos. Os efeitos das diferentes densidades de plantas foram mais nítidos nos componentes da produção, em que 
Tabela 3 - Número de perfilhos por planta, de espigas por metro e de espiguetas por espiga do trigo em função da densidade de plantas, de doses de nitrogênio e do regulador de crescimento trinexapac-ethyl. Fazenda Escola, UEPG. Ponta Grossa, PR. 1999.

\begin{tabular}{|c|c|c|c|}
\hline $\begin{array}{l}\text { Densidade } \\
\text { (plantas/m) }\end{array}$ & $\begin{array}{l}\text { Perfilho/planta } \\
\text { Média }\end{array}$ & $\begin{array}{l}\text { Espigas/m } \\
\text { Média }\end{array}$ & $\begin{array}{c}\text { Espiguet./espiga } \\
\text { Média }\end{array}$ \\
\hline 55 & $2,45 \mathrm{a}$ & $85,1 \mathrm{c}$ & $12,9 \mathrm{a}$ \\
\hline 75 & $2,12 b$ & $98,6 \mathrm{~b}$ & $12,5 \mathrm{~b}$ \\
\hline 112 & $1,37 \mathrm{c}$ & $108,4 a$ & $11,8 \mathrm{c}$ \\
\hline Nitrogênio $(\mathrm{kg} / \mathrm{ha})$ & Média & Média & Média \\
\hline 0 & --- & $87,6 \mathrm{c}$ & $12,5 \mathrm{a}$ \\
\hline 45 & --- & $96,4 \mathrm{~b}$ & $12,3 \mathrm{a}$ \\
\hline 90 & --- & $101,6 a b$ & $12,3 a$ \\
\hline 135 & --- & $103,8 \mathrm{a}$ & $12,4 \mathrm{a}$ \\
\hline Média & Com & Com & Com \\
\hline Trinexapac-ethyl & $6,1 \mathrm{~B} \quad 7,5 \mathrm{~A}$ & $12,1 \mathrm{~B} \quad 16,8 \mathrm{~A}$ & $18,0 \mathrm{~B} \quad 19,0 \mathrm{~A}$ \\
\hline Coef. variação & $7,0 \%$ & $11,1 \%$ & $5,5 \%$ \\
\hline
\end{tabular}

Médias seguidas da mesma letra, minúsculas nas colunas e maiúsculas na linhas, não diferem pelo teste da DMS (t teste) ao nível de 5\% de probabilidade.

o número de espigas por metro aumentou com o aumento da densidade (Tabela 3). Entretanto, as plantas não mostraram potencial para o enchimento dos grãos e, assim, o número de espiguetas por espiga (Tabela 3) e o peso de 1000 grãos (Tabela 4) diminuíram com o aumento da densidade, adequando o número e o peso dos grãos às

Tabela 4 - Peso de 1000 grãos (g) e produtividade de grãos (kg/ha) do trigo em função da densidade de plantas, de doses de nitrogênio e do regulador de crescimento trinexapac-ethyl. Fazenda Escola, UEPG. Ponta Grossa, PR. 1999.

\begin{tabular}{|c|c|c|c|c|}
\hline $\begin{array}{l}\text { Densidade } \\
\text { (plantas/m) }\end{array}$ & \multicolumn{2}{|c|}{$\begin{array}{l}\text { Peso de grãos } \\
\text { Média }\end{array}$} & \multicolumn{2}{|c|}{$\begin{array}{c}\text { Produtividade } \\
\text { Média }\end{array}$} \\
\hline 55 & \multicolumn{2}{|c|}{$38,5 \mathrm{a}$} & \multicolumn{2}{|c|}{$2.351 \mathrm{a}$} \\
\hline 75 & \multicolumn{2}{|c|}{$37,0 \mathrm{~b}$} & \multicolumn{2}{|c|}{$2.247 \mathrm{a}$} \\
\hline 112 & \multicolumn{2}{|c|}{35,9 b } & \multicolumn{2}{|c|}{$2.241 \mathrm{a}$} \\
\hline Nitrogênio $(\mathrm{kg} / \mathrm{ha})$ & \multicolumn{2}{|c|}{ Média } & \multicolumn{2}{|c|}{ Média } \\
\hline 0 & \multicolumn{2}{|c|}{$37,5 \mathrm{a}$} & \multicolumn{2}{|c|}{$1.746 \mathrm{c}$} \\
\hline 45 & \multicolumn{2}{|c|}{$36,9 a$} & \multicolumn{2}{|c|}{$2.246 \mathrm{~b}$} \\
\hline 90 & \multicolumn{2}{|c|}{$37,5 \mathrm{a}$} & \multicolumn{2}{|c|}{$2.505 \mathrm{a}$} \\
\hline 135 & \multicolumn{2}{|c|}{$36,6 a$} & \multicolumn{2}{|c|}{$2.623 a$} \\
\hline Média & Com & Sem & Com & Sem \\
\hline Trinexapac-ethyl & $35,6 \mathrm{~B}$ & $38,6 \mathrm{~A}$ & $2.544 \mathrm{~A}$ & $2.016 \mathrm{~B}$ \\
\hline Coef. variação & \multicolumn{2}{|c|}{$5,9 \%$} & \multicolumn{2}{|c|}{$11,4 \%$} \\
\hline
\end{tabular}

Médias seguidas da mesma letra, minúsculas nas colunas e maiúsculas na linhas, não diferem pelo teste da DMS (t teste) ao nível de $5 \%$ de probabilidade. condições do ambiente, fato que resultou em produtividade similar para as densidades estudadas. Considerando que as densidades estudadas correspondem a 323, 441 e 658 plantas por metro quadrado e, segundo MUNDSTOCK (1983) entre 180 e 570 plantas por metro quadrado, a produtividade varia muito pouco face ao perfilhamento da cultura, a similaridade dos resultados no presente trabalho, pode ser considerada normal para as densidades utilizadas, corroborando com os resultados obtidos por SILVA \& GOMES (1990), que não observaram diferenças na produtividade para densidades variáveis entre 200 e 400 plantas $/ \mathrm{m}$.

$$
\text { A aplicação do nitrogênio, }
$$
independente da dose, promoveu aumento do comprimento do terceiro e do quarto entre-nós, aqueles visíveis na época da aplicação do nutriente. Para o segundo entre-nó, aquele que emergiu após o trinexapac-ethyl já estar atuando na planta, os efeitos foram mais nítidos, ocorrendo uma redução substancial do comprimento para as doses 0 e $45 \mathrm{~kg} /$ ha de nitrogênio, somente quando o trinexapac-ethyl foi aplicado. Para as maiores doses, o comprimento dos entre-nó foi maior, porém, ainda menor daquele observado quando da não aplicação do produto. O primeiro entre-nó não foi afetado pela aplicação de nitrogênio.

A estatura de plantas (Tabela 2) aumentou com o aumento da dose de nitrogênio para os tratamentos com trinexapac-ethyl e, mesmo na maior dose, a altura foi menor daquela observada na ausência do produto, em que a dose de nitrogênio não afetou esta característica. A redução da altura de plantas aparece como uma das vantagens mais importantes do trinexapac-ethyl e sua interação com a dose de nitrogênio permite o uso de doses maiores do nutriente, mantendo as plantas com altura inferior àquela observada na ausência do produto. Como a adição de doses nitrogênio a partir de $40 \mathrm{~kg} / \mathrm{ha}$ já implica no acamamento (SILVA \& GOTO, 1991) e em geral são utilizadas doses de até $120 \mathrm{~kg} / \mathrm{ha}$ com bons resultados (FREITAS et al., 1994), para as cultivares de porte médio e alto e/ou em locais sujeitos a ocorrência de ventos, o trinexapac-ethyl pode constituir uma opção para o aumento da produtividade, por possibilitar o uso de maiores doses de nitrogênio sem a ocorrência de acamamento.

O diâmetro do caule e a massa das plantas secas (Tabela 2) não foram afetados pela adição ou não de nitrogênio. A produtividade de grãos (Tabela 4) aumentou com o aumento da dose de nitrogênio até $90 \mathrm{~kg} / \mathrm{ha}$, que foi função do aumento do número de espigas por metro, uma vez que o número de 
espiguetas por espiga e o peso de 1000 grãos não foram afetados pela aplicação do nutriente. FREITAS et al. (1995) verificaram aumento crescente de produtividade com o aumento da dose de nitrogênio de 0 até $120 \mathrm{~kg} / \mathrm{ha}$ para a média de oito cultivares e que confirmam o potencial do trigo em responder a altas doses de nitrogênio. Entre as doses de 90 e $120 \mathrm{~kg} / \mathrm{há}$, não foram observadas diferenças em nenhum dos fatores avaliados, indicando ser desnecessário o uso da segunda para a cultivar em estudo.

\section{CONCLUSÕES}

A aplicação do trinexapac-ethyl resultou em plantas com entre-nós mais curtos; em aumento do número de espigas/m e da produtividade.

Com o aumento da dose de nitrogênio ocorreu aumento da estatura das plantas, do número de espigas $/ \mathrm{m}$ e da produtividade.

Com o aumento da densidade de plantas, o diâmetro do caule, a massa seca das plantas e o número de grãos por espiga diminuíram, e o número de espigas/m e o peso de mil grãos aumentaram, mas não afetaram a produtividade.

\section{REFERÊNCIAS BIBLIOGRÁFICAS}

AMREIN, J.; RUFENER, M.; QUADRANTI, M. The use of CGA $163^{\prime} 935$ as a growth regulator in cereals and oilseed rape. In: BRIGHTON CROP PROTECTION CONFERENCE - WEEDS, 1989. Switzerland. Proceedings... Switzerland : Ciba Geigy, 1989. p.2-12.

CAVASSIM, J.E.; BORÉM, A. Correlações em seis populações de trigo (Triticum aestivum L.). Revista Ceres, v.45, n.262, 1998. p.555-566

COSTA, J.M.; OLIVEIRA, E.F. Fertilidade do solo e nutrição de plantas. Campo Mourão, PR: COAMO/CODETEC, 1998. $89 \mathrm{p}$

DAVIES, P.J. The plant hormones: their nature, occurrence and functions. In: DAVIES, P.J. Plant hormones and their role in plant growth and development. Netherlands : Kluwer Academic, 1987. p.1-23.

EMBRAPA. Cultivares de trigo do Paraná. Londrina : Embrapa/CNPSo, 1997. 148p. (EMBRAPA-CNPSo. Circular Técnica, 18).
FREITAS, J.G.; CAMARGO, C.E.O.; PEREIRA FILHO, A.W.P., et al. Produtividade e resposta de genótipos de trigo ao nitrogênio. Bragantia, Campinas. v.53, n.2, p.281-290, 1994..

FREITAS, J.G.; CAMARGO, C.E.O.; PEREIRA FILHO, A.W.P., et al. Eficiência e resposta de genótipos de trigo ao nitrogênio. Revista Bras da Ciência do Solo, Campinas, v.19, p.229-234, 1995.

GE, Y.X.; ZHANG, S.Z. Studies on the ideotype of wheat. II. Studies on the relationship among characters of different stature series of wheat. Acta Agriculturae Universitatis Pekinensis, v.16, p.343-351, 1990.

IAPAR. Informações técnicas para a cultura de trigo no Paraná - 1999. Londrina, 1999. 148p. (IAPAR, Circular, 106).

KERBER, E.; LEYPOLD, G.; SEILER, A. CGA 163'935 a new plant growth regulator for small grain cereals, rape and turf. In: BRIGHTON CROP PROTECTION CONFERENCE WEEDS, 1989, Switzerland. Proceedings... Switzerland : Ciba Geigy, 1989. p.83-88.

MUNDSTOCK, C.M. Cultivo dos cereais de estação fria: trigo, cevada, aveia, centeio, alpiste, triticale. Porto Alegre : NBS, 1983. $265 \mathrm{p}$.

MUNDSTOCK, C.M. Planejamento e manejo integrado da lavoura de trigo. Porto Alegre : ed. do Autor, 1999. 228p.

SEGANFREDO, R. Seleção de variedade de trigo para o ano agrícola de 1999. Informativo Fundação ABC, v.1, n.2, p.16-17, 1999.

SILVA, D.B.; GOMES, A.C. Espaçamento e densidade de semeadura em trigo irrigado na região dos cerrados. Pesq Agrop Bras., Brasília, v.25, n.3, p.305-315, 1990.

SILVA, D.B.; GOTO, W.S. Resposta do trigo de sequeiro ao nitrogênio, após soja precoce, na região do Alto Paranaíba, MG. Pesq Agrop Bras, Brasília, v.26, n.9, p.1401-1405, 1990.

VIEIRA, R.D.; FORNASIERI FILHO, D.; MINOHARA, L., $\boldsymbol{e}$ al. Efeito de doses e épocas de aplicação de nitrogênio em cobertura na produção e na qualidade físiológica de sementes de trigo. Científica, v.23, n.2, p.257-264, 1995.

WEILER, E.W.; ADAMS, R. Studies on the action of the new growth retardant CGA 163'935. In: BRIGHTON CROP PROTECTION CONFERENCE - WEEDS, 1991, Switzerland. Proceedings... Switzerland : Ciba Geigy, 1991. p.1133-1138. 